\title{
BYOE: A Method for Creating CAD-based, Two-sided Molded Prototype Parts
}

\section{Dr. Christopher P. Pung, Grand Valley State University}

Dr. Pung has interests in experiential learning, design processes and student teams.

\section{Amanda E. Stark, NASA Langley Research Center}

Amanda E. Stark received her Bachelor of Fine Art in Art and Design (2003) from Grand Valley State University and a Master of Fine Art (2006) from Bowling Green State in 3-Dimensional Art. Her art work has received numerous awards and has been added to some permanent collections. She has a Bachelor of Science in Engineering (2016) from Grand Valley State University. She is the recipient of the Wisner Engineering Scholarship (2015-2016) and two NASA NIFS Internships (2015). She is currently pursuing a Master of Science in Mechanical Engineering at Old Dominion University and has been awarded a Pathways Engineering Trainee Position at NASA Langley Research Center in Hampton, Virginia.

\section{Mr. Matthew Steven Dykstra}

Jill Morris 


\section{BYOE: A Method for Creating CAD-based Two-sided Molded Prototype Parts}

Pedagogical motivation: At Grand Valley State University senior engineering students in a product design class have struggled with internalizing concepts relating to designing for injection molding. Past projects for the class have resulted in designs for components that are intended for injection molding. However, the final designs have included parts that have features not compatible with the injection molding process or stated limits on the complexity of the mold. These incompatibilities have included: lack of or insufficient draft to allow the part to release from the mold, the use of multiple cores, collapsing cores, mold lock and others. The students that engaged in the hands-on prototype creation detailed below produced designs that were compatible with an injection molding process.

Introduction:

As technology advances, injection molding is proving to be a viable production method for silicone parts. Injection molding has many advantages over other manufacturing processes such as machining. Injection molding allows for a variety of silicone part complexities to be produced in approximately the same amount of processing time. Additionally, the silicone parts that are produced are near net shape and require minimal additional processing afterwards. The downfall of injection molding is the high cost associated with producing molds and maintaining the machine. Steel molds can range in cost from $\$ 6,000$ to $\$ 250,000+($ Cost Estimator, 2017) and more, depending on geometry, finish, and tolerances that are applied to the mold. During the prototyping phase of a product the product cost associated with machining molds for the part for each revision is not economically feasible. This leads to last minute design revisions that can be detrimental to the project timeline when the final prototype does not meet the required requirements. One method that is currently used to reduce the cost of prototypes is the use of different mold materials. Aluminum molds are capable of maintaining dimensional integrity of the part for a short life. The aluminum molds can range in cost from $\$ 1,000$ to $\$ 5,000$, depending on geometry, finish, and tolerances that are applied to the mold. While this is a cost effective alternative to steel molds for prototypes, the cost of maintaining the injection molding machine is still significant. Another alternative to steel and aluminum molds is to utilize additive 
manufacturing processes to produce plastic molds. Built-in features of CAD packages allow these molds to modelled and then exported to rapid prototype machines. These molds do not possess the structure of metal molds but are sufficient for casting silicone parts. This process allows for fast feedback on design functionality while minimizing cost and tooling production time.

Required Materials:

The following materials where used for the casting process:

1. 3-D extrusion printer, MakerBot Replicator 2

2. $1.75 \mathrm{~mm}$ MakerBot PLA filament

3. SolidWorks 2014, Educational Edition

a. Mold Tools Add-In

4. DAP $100 \%$ silicone caulk

5. Argo $100 \%$ Corn Starch

6. Rubber Bands

7. Balance

8. Mixing plate and utensils

9. PAM no-stick cooking spray

10. Dawn dish soap

Process:

The process of casting prototype silicone parts was divided into four portions; 1) generating part geometry, 2) generating mold geometry, 3) manufacturing the mold, 4) producing parts using the mold. For the purposes of this paper, it is assumed that a part CAD model exists. Additionally, the steps shown below are for SolidWorks 2014, Educational Edition with the Mold Tools AddIn. Newer versions should function the same way, however slight alterations to the process steps may be needed.

Part Geometry Lessons Learned:

The wall thickness of the part should be less than or equal to ten $\mathrm{mm}$. If the wall thickness is greater than ten $\mathrm{mm}$, allow for extra curing time or consider an alternative prototyping process. Similar to an injection molded part, draft is desired in part to allow for ease of part removal. 
Through iteration, it was discovered that a draft angle between half a degree and three degrees produced useful parts. Many CAD packages have surfacing or sheet metal handling packages. Utilizing these tools may help avoid thick sections.

Generating Mold Geometry:

With the prototype part open in SolidWorks, ensure the Mold Tools Add-In is added and is running.

Parting Line:

The parting line is the line that will separate the core and the cavity sections of the mold. To generate this line, start by selecting Parting Lines on the Mold Tools tab shown in Figure 1.

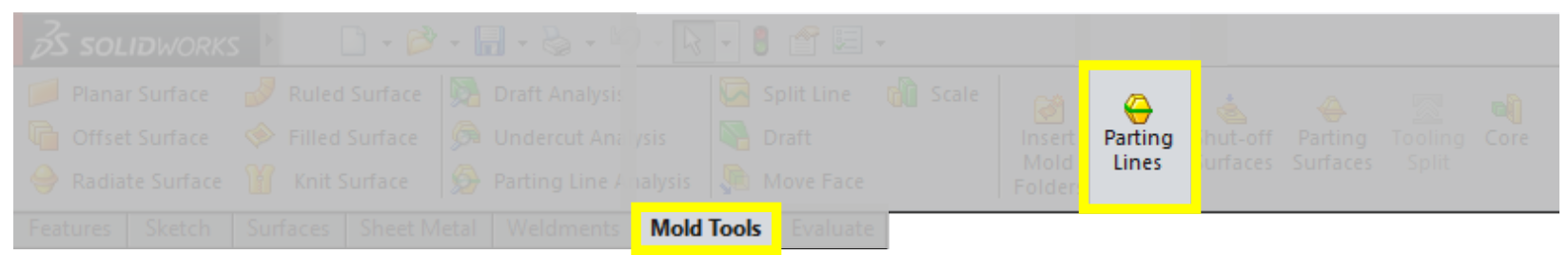

Figure 1: Parting line tool location in SolidWorks 2014, education edition.

Within the Parting Lines tool, select the plane which the two mold halves will be split about under the mold parameters. Enter the minimum draft angle for the part and select draft analysis, shown in Figure 2. 


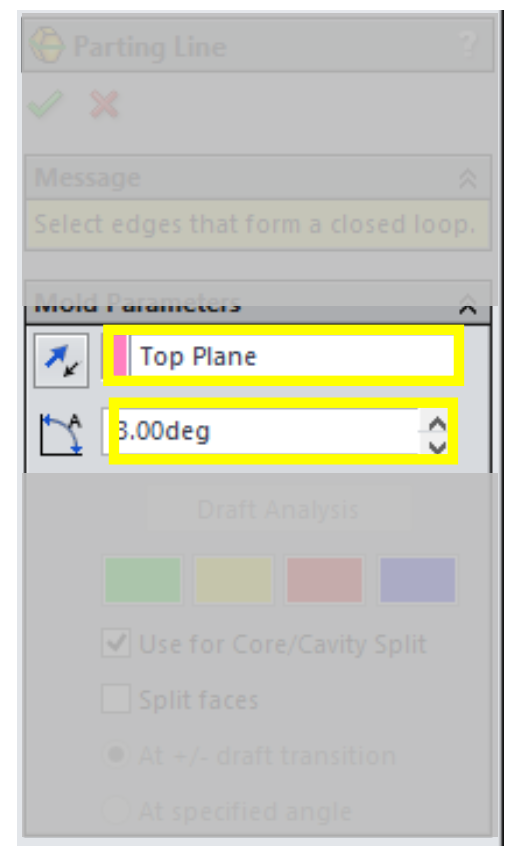

Figure 2: Selection for draft angle analysis and parting line direction in SolidWorks 2014, education edition.

Inspect the model looking at the draft analysis that has been performed. The model should have positive draft surface on the core surfaces, represented by a green surface. On the cavity surfaces, the part should have a negative draft, represented by a red surface. If a core surface has a negative draft or a cavity surface has a positive draft, the part should be reconfigured (orientation or design) or use molds with more than two parts. If any surface on the design has zero draft, represented by yellow, or is a straddle surface, represented by blue, the model should be reconfigured if possible to minimize these features.

When the draft analysis is satisfactory, the parting line is ready to be selected. Select the edge that separates the positive draft angles from the negative draft angles. If parting line is complex, reevaluate part geometry to simplify. When satisfied with the parting line, select the check mark to complete the process. Figure 3 illustrates the parting line for a part. 


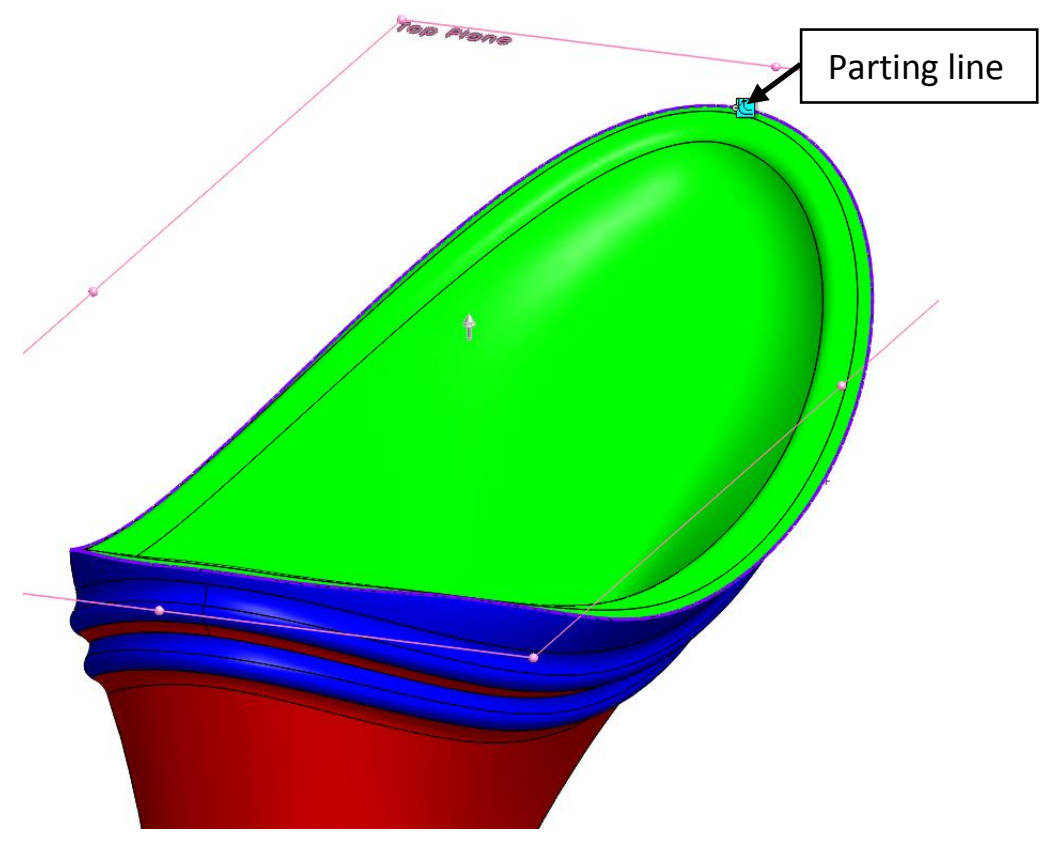

Figure 3: Illustration of parting line selection and draft angle analysis in SolidWorks 2014, education edition.

Shut-off Surfaces:

Shut-off surfaces are used to produce open holes in the final part. To generate these start by selection Shut-off Surfaces on the Mold Tools tab.

Within the Shut-off Surfaces menu, we will select the edges of open holes in the mold. This will generate the surface to close the model for when the mold is made as shown in Figure 4.

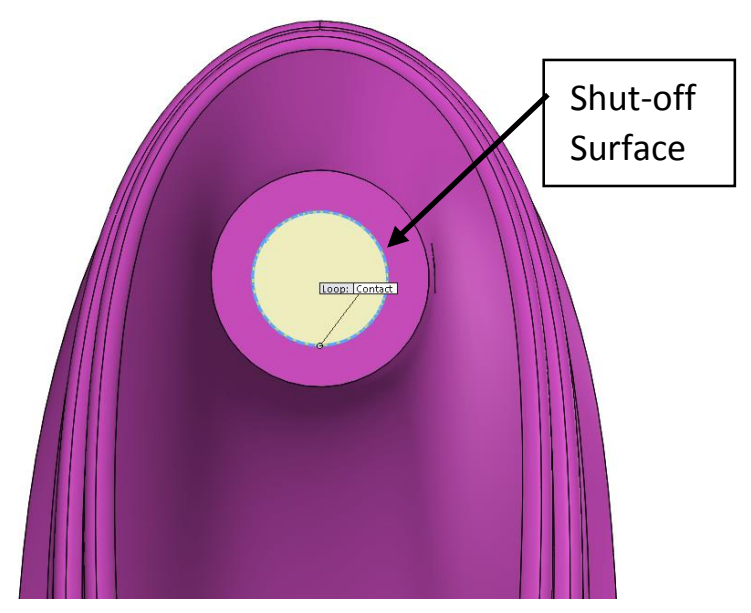

Figure 4: Illustration of shut-off surface in SolidWorks 2014, education edition. 
When all of the shut-off surfaces have been defined verify that they have built properly within the model. When the surfaces are built properly, press the check mark. With the shut-off surfaces defined, the next step is to define the parting surface.

\section{Parting Surface:}

The parting surface is the surface that will separate the cavity and the core portions of the mold. To generate these start by selection Parting Surface on the Mold Tools tab.

Within the Parting Surface menu, the parting line that was previously generated will be selected, if not, expand the design tree within the sketch window and select the appropriate parting line. Ensure that that parting surface is extending outwards away from the part. Additionally, adjust the distance to allow for the parting surface to extend beyond the edges of the mold body that we will make in a later step. For a small part, an extension of $30 \mathrm{~mm}$ is adequate. If an error occurs during the parting surface generation the parting line geometry may need to be altered. Figure 5 illustrates the part geometry and menu selection in SolidWorks.
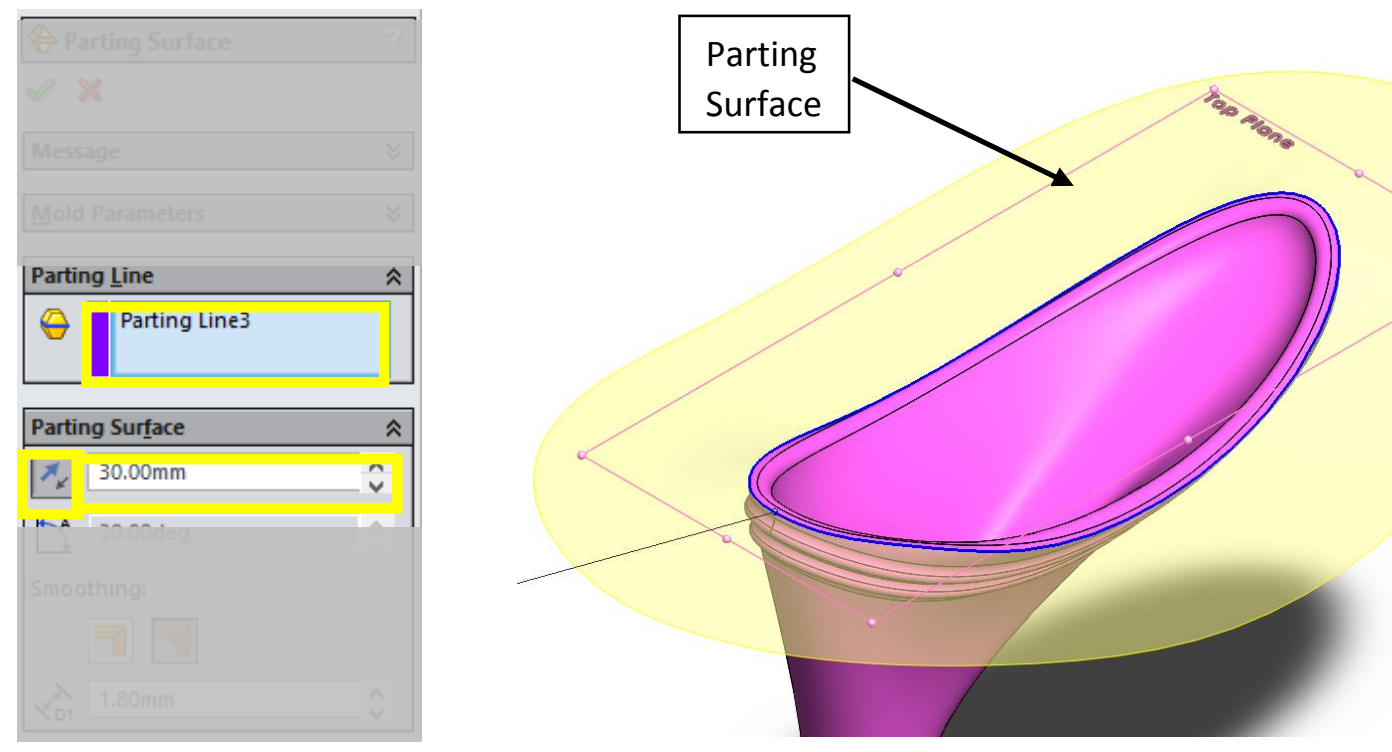

Figure 5: Selection of parting line and sizing along with illustration in SolidWorks 2014, education edition. 


\section{Tooling Split:}

The tooling split generates the bodies that will be the cavity and the core portions of the mold. To generate these start by selection Tooling Split on the Mold Tools tab. To start the tooling split process, a sketch must be drawn to represent the outer surfaces of the mold. When tooling split is selected, this sketch process is automatically started; select the plane that was selected for the parting line to sketch your mold. For simple molds, a rectangle is adequate to encapsulate the part. The sketch must fully encapsulate the model, but not extend past the parting surface. With the sketch defined, the next step is to define the depth of the mold halves. Use the inputs in the feature manager or drag and drop the arrows to the desired points on the sketch plane. The depth of the mold must fully encapsulate the model. When all of the above is satisfied, press the check mark. Figure 6 shows the selection of the mold bounding box in SolidWorks.

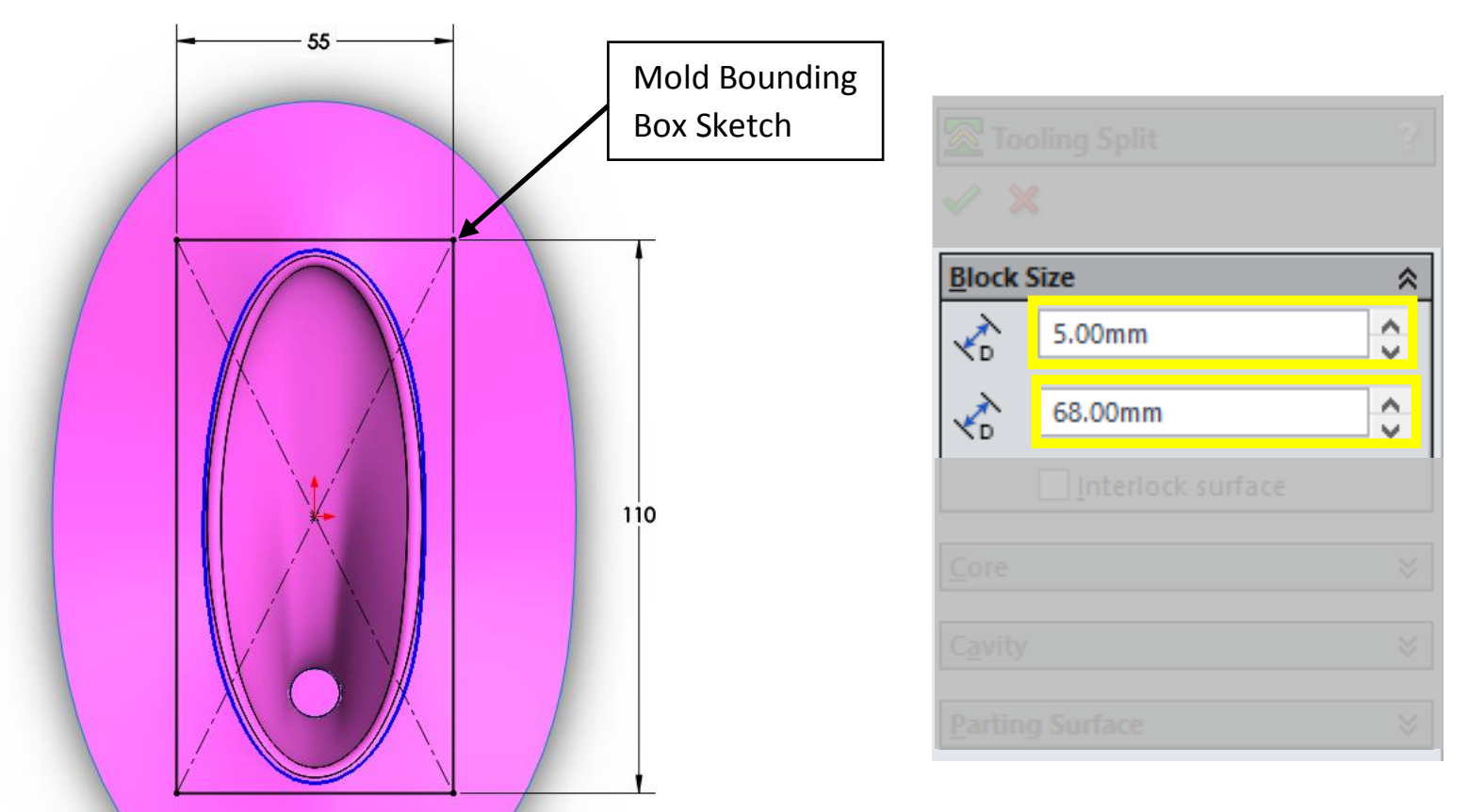




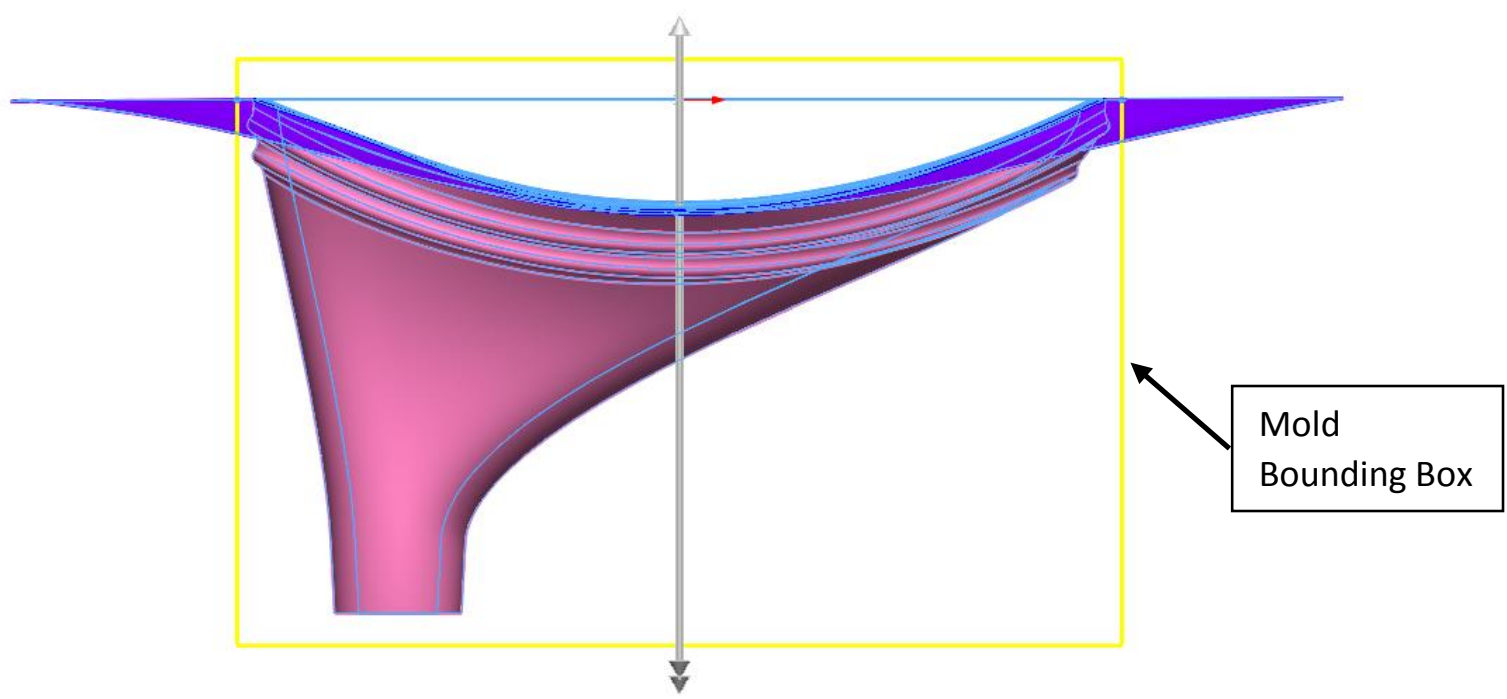

Figure 6: Selection of mold size with illustrations in SolidWorks 2014, education edition.

With the tooling split complete, the mold haves are now ready to be isolated into separate parts.

\section{Part Isolation:}

To generate models of the two mold halves, they must be inserted into new parts. Use the feature tree and expand the Solid Bodies Folder as shown in Figure 7. Right click on "Tooling Split[1]", select "Insert into New Part"; press the check mark on the feature manager. Save the new mold with an appropriate name and repeat process for the other mold halve. The mold is now ready to be manufactured using a 3-D printer. 


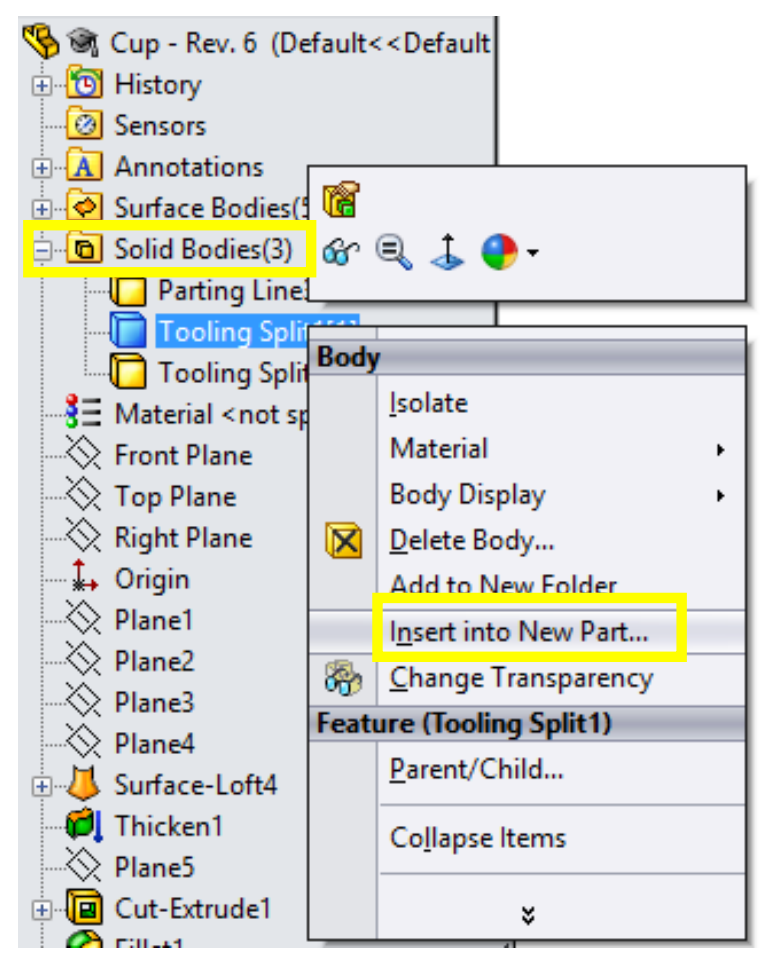

Figure 7: Insertion of mold half into a new part in in SolidWorks 2014, education edition.

Creating the Molds:

Open the mold files in SolidWorks. Save each of the mold halves as an STL file. These files will be used to generate the machine path in the 3-D printer's software.

\section{3-D Printing:}

Open the .STL files for the mold halves in the 3-D printer's software, positioning the parts so the core and cavity surfaces are facing upwards on the build plate. To determine the properties that must be used for the print, the complexity of the part as well as the quality of the part to be printed must be considered. For parts that has many complex surfaces or a smoother surface finish is desired, a high build quality should be used. Conversely, for simple parts or parts where the surface finish is important, a low quality build should be used. Use the information provided in Table 1 to determine the correct print settings. 
Table 1: 3-D printing recommended settings for casting molds.

\begin{tabular}{|c|c|c|c|c|c|c|}
\hline Quality & $\begin{array}{c}\text { Layer } \\
\text { Height }\end{array}$ & $\begin{array}{c}\text { Number } \\
\text { of Shells }\end{array}$ & Raft & Support & Material & $\begin{array}{c}\text { Extruder } \\
\text { Temperature }\end{array}$ \\
\hline High & $0.10 \mathrm{~mm}$ & 2 & Yes & Yes & $\begin{array}{c}1.75 \mathrm{~mm} \\
\text { PLA }\end{array}$ & $220^{\circ} \mathrm{C}$ \\
\hline Low & $0.20 \mathrm{~mm}$ & 2 & Yes & No & $\begin{array}{c}1.75 \mathrm{~mm} \\
\text { PLA }\end{array}$ & $220^{\circ} \mathrm{C}$ \\
\hline
\end{tabular}

The printed molds should be sanded with 220 grit sand paper to help increase the surface finish of the final product. To further enhance the surface finish of the molds, a filling primer paint can be sprayed onto the mold and sanded (PlastiKote ${ }^{\mathrm{TM}}$ primer was used successfully).

Producing Parts:

\section{Prep Work:}

Spray the surface of the mold with no-stick cooking spray. The cooking spray serves as a mold release agent that will help with part removal after the casting has cured. For the parts shown, PAM cooking spray was used.

Casting Material: A mixture of silicone and cornstarch was used to produce parts. Depending on the desired stiffness of the part, the ratio of silicone to cornstarch can be changed. Parts were created using a variety of ratios. The ratios producing usable parts ranged from 1:1 silicone to cornstarch by weight to 1:6. A design of experiments was conducted which included this ratio as a variable is included in appendix A. The increased amount of cornstarch yielded stiffer parts. In addition to stiffening the silicone, the corn starch also acts as a secondary mold release agent. Food or egg dye was used successfully to create colored parts. Figure 8 shows the cornstarch and silicone mixture. 

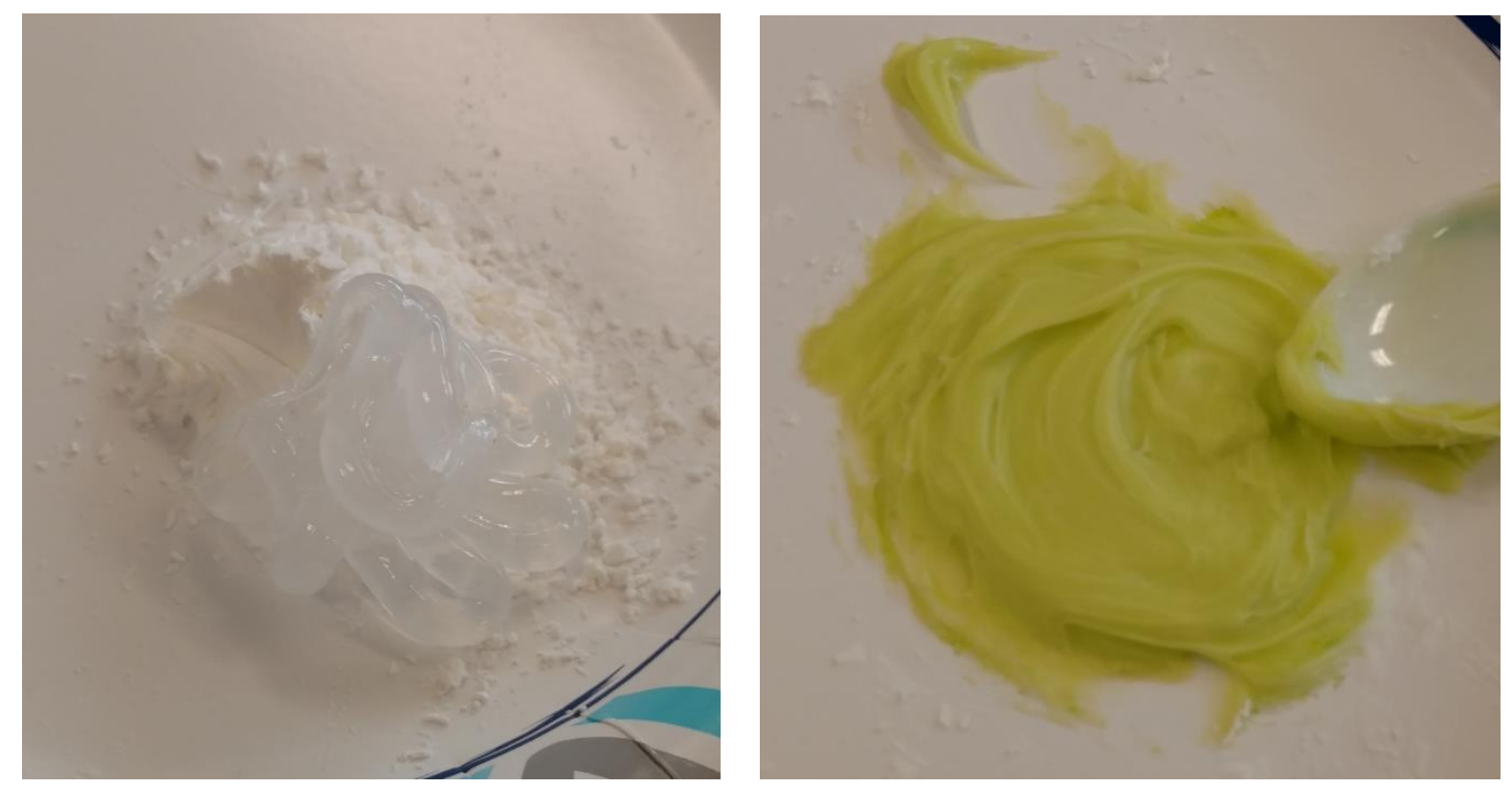

Figure 8: Silicone and cornstarch before and after mixing. Yellow food was added for aesthetics.

\section{Casting Parts:}

When the material has been thoroughly mixed is should be added immediately to the cavity mold. Ensure that the material is spread throughout the mold to minimize the number of voids I the casting. Figure 9 shows a filled mold.

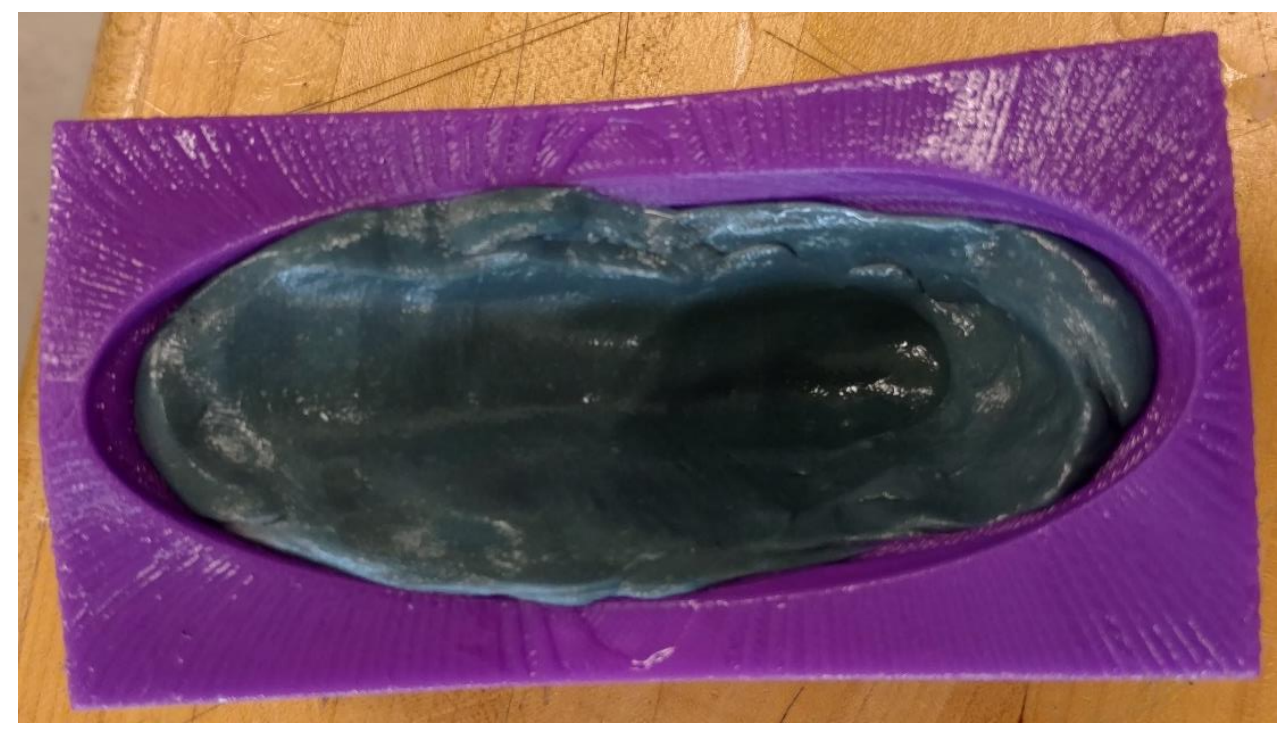

Figure 9: Insertion of silicone into mold, ensure the material is spread evenly with no holes. 
When the material has been added to the cavity, the core side of the mold is ready to be inserted into the cavity mold. Using your hands, squeeze the mold to achieve the proper setting. Place the assembled mold onto a table and use a heavy object, such as a barbell weight or a large book to keep pressure on the two halves of the mold during the curing process as shown in Figure 10.

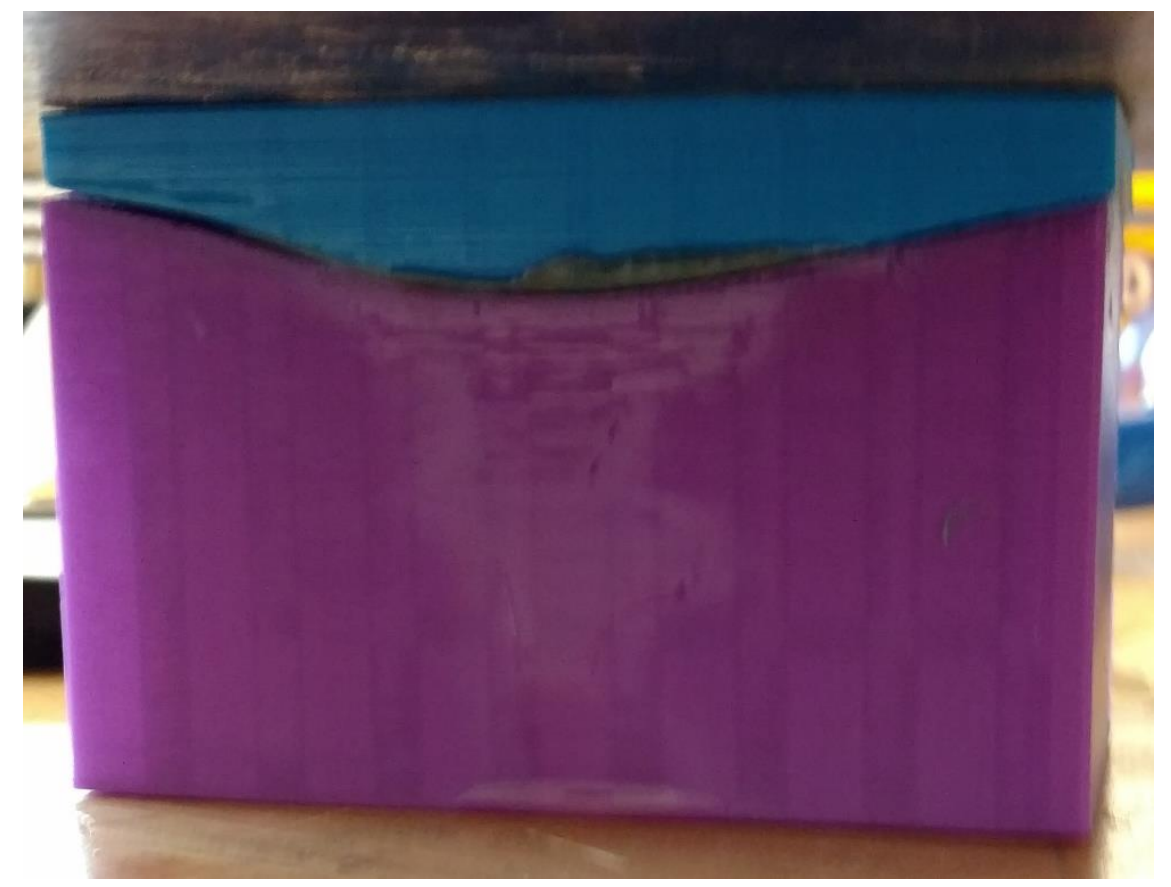

Figure 10: Completed mold with weight on top, allow at least 12 hours to cure.

Allow 12 hours for the silicone to fully cure before removing the weight from the mold. When the curing time has passed, use a small screw driver to pry the mold halves apart at the parting surface. Carefully remove the silicone casting from the core surface of mold and trim the flash from it. Wash the part using Dawn dish soap or an equivalent. Thicker sections are easily achieved using spacers between the mold halves. If voids are a concern the assembly can be vacuumed bagged.

Cost Estimator. (2017, 3 21). Retrieved from CustomPart.net: http://www.custompartnet.com/estimate/injection-tooling/ 
Appendix A - Summary of a Design of Experiments for Silicone Casting

Four variables were included in the design of experiments they are listed in Table 2 along with the levels.

Table 2. Silicone Casting Variables and Levels.

\begin{tabular}{l|ccc} 
Variable & Low $(-1)$ & Middle $(0)$ & High (1) \\
\hline $\begin{array}{l}\text { Silicone-Cornstarch Ratio } \\
\text { (mass) }\end{array}$ & $3: 1$ & $2: 1$ & $1: 1$ \\
Dish Soap & & & \\
Thickness (mm) & none & 1 tbsp/1000ml & 2tbsp/1000ml \\
Silicone Caulk Color & 1.5 & 2.5 & 3.5 \\
& White & Clear & Silver
\end{tabular}

The measured response was durometer using the Shore A scale. A Box and Behken style design of experiments was used. Table 3 lists the experimental runs and response.

Table 3 Experimental Runs and Response

\begin{tabular}{|c|c|c|c|c|c|c|c|c|c|}
\hline+ & C1 & C2 & C3 & $\mathrm{C} 4$ & $\mathrm{C} 5$ & C6 & C7 & $\mathrm{C} 8$ & $\mathrm{C} 9$ \\
\hline & StdOrder & RunOrder & PtType & Blocks & Ratio & Color & Thickness & Soap & Durometer \\
\hline 1 & 8 & 1 & 2 & 1 & 0 & 0 & 1 & 1 & 24.33 \\
\hline 2 & 4 & 2 & 2 & 1 & 1 & 1 & 0 & 0 & 40.33 \\
\hline 3 & 19 & 3 & 2 & 1 & -1 & 0 & 1 & 0 & 23.00 \\
\hline 4 & 11 & 4 & 2 & 1 & -1 & 0 & 0 & 1 & 22.00 \\
\hline 5 & 9 & 5 & 2 & 1 & -1 & 0 & 0 & -1 & 21.00 \\
\hline 6 & 25 & 6 & 0 & 1 & 0 & 0 & 0 & 0 & 28.33 \\
\hline 7 & 3 & 7 & 2 & 1 & -1 & 1 & 0 & 0 & 21.67 \\
\hline 8 & 12 & 8 & 2 & 1 & 1 & 0 & 0 & 1 & 38.67 \\
\hline 9 & 16 & 9 & 2 & 1 & 0 & 1 & 1 & 0 & 26.00 \\
\hline 10 & 15 & 10 & 2 & 1 & 0 & -1 & 1 & 0 & 25.17 \\
\hline 11 & 1 & 11 & 2 & 1 & -1 & -1 & 0 & 0 & 18.00 \\
\hline 12 & 6 & 12 & 2 & 1 & 0 & 0 & 1 & -1 & 23.67 \\
\hline 13 & 24 & 13 & 2 & 1 & 0 & 1 & 0 & 1 & 27.33 \\
\hline 14 & 23 & 14 & 2 & 1 & 0 & -1 & 0 & 1 & 27.00 \\
\hline 15 & 21 & 15 & 2 & 1 & 0 & -1 & 0 & -1 & 25.00 \\
\hline 16 & 27 & 16 & 0 & 1 & 0 & 0 & 0 & 0 & 23.17 \\
\hline 17 & 13 & 17 & 2 & 1 & 0 & -1 & -1 & 0 & 31.67 \\
\hline 18 & 20 & 18 & 2 & 1 & 1 & 0 & 1 & 0 & 38.17 \\
\hline 19 & 2 & 19 & 2 & 1 & 1 & -1 & 0 & 0 & 35.00 \\
\hline 20 & 10 & 20 & 2 & 1 & 1 & 0 & 0 & -1 & 34.50 \\
\hline 21 & 26 & 21 & 0 & 1 & 0 & 0 & 0 & 0 & 23.00 \\
\hline 22 & 7 & 22 & 2 & 1 & 0 & 0 & -1 & 1 & 31.67 \\
\hline 23 & 5 & 23 & 2 & 1 & 0 & 0 & -1 & -1 & 30.67 \\
\hline 24 & 17 & 24 & 2 & 1 & -1 & 0 & -1 & 0 & 31.67 \\
\hline 25 & 18 & 25 & 2 & 1 & 1 & 0 & -1 & 0 & 42.17 \\
\hline 26 & 22 & 26 & 2 & 1 & 0 & 1 & 0 & -1 & 28.50 \\
\hline 27 & 14 & 27 & 2 & 1 & 0 & 1 & -1 & 0 & 38.33 \\
\hline
\end{tabular}

